\title{
Presencia y difusión de contenidos de las televisiones universitarias españolas en la web
}

\author{
María Murillo Guerrero
}

Jorge Caldera-Serrano

Recibido: 14 de octubre de 2014

Aceptado: 10 de noviembre de 2014

\section{Resumen}

Se examina la existencia de televisiones educativas en las universidades españolas, estudiándose los elementos y contenidos que presentan en su caso. Se parte del estado de la cuestión de las televisiones educativas. Se realiza un análisis de las web universitarias y de las web televisivas a partir de una ficha tipo que presenta elementos de carácter formal y de contenido. De esos datos se extraen los resultados a modo de gráficos, y de ellos las conclusiones del estudio y la validación o refutación de las hipótesis a priori planteadas. Este trabajo es el resultado del proceso de investigación en el marco del Trabajo Fin de Máster del programa de "Gestión de la Información Digital".

\section{Palabras claves}

Contenidos difundidos, Televisión, Televisión educativa, Televisión online, Televisión Universitaria; Universidades españolas.

\section{Presence and broadcasting of the contents of spanish university televisión on the web}

\section{Abstract}

Existence of educational television in the Spanish universities is analysed, considering their main elements and contents. Starting from the condition of educational television, university and television websites are tested through a datasheet in which formal and content facts are considered. Graphic charts are generated from those results, and so are conclusions and refutations related to the initial hypothesis. This labour is the result of the investigation process linked to the Master's Thesis of the "Digital Information Management" program.

\section{Key words}

Educational Television, Online Television, Spanish Universities, Speeded contents, Television, University Television

http://dx.doi.org/10.5209/rev CDMU.2014.v25.47475

cuadernos ae vocumentacion iviulumeald vol. $\angle$ J. 


\section{TELEVISIONES UNIVERSITARIAS}

La televisión se ha mostrado como un gran desarrollo tecnológico y comunicacional. Se ha demostrado su gran capacidad para llegar a grandes masas eliminando los impedimentos relacionados con el espacio. La televisión ha logrado convertirnos en una auténtica "aldea global", donde todos compartimos la misma información, el mismo entretenimiento y, en definitiva, el mismo modelo de vida.

Herramienta informativa, comunicativa, educativa, publicista, etc., la televisión ha sido utilizada con muchos fines y por diversos motivos. La televisión ha inundado todos y cada uno de los ámbitos de la vida. La televisión es parte de nuestra realidad, es parte de nuestra idiosincrasia, la columna vertebral de nuestra comunidad.

En la actualidad, esa hegemonía de la televisión tradicional como elemento de comunicación está comenzando a contar con algunos adversarios. La aparición de internet y el notable desarrollo actual de las redes sociales hacen que los nativos digitales cuenten con otras fórmulas para conseguir informarse, formarse o entretenerse. Es por este motivo que las televisiones buscan nuevas formas de comunicación con las que adaptarse a esta realidad cambiante. Es debido a este avance en las nuevas tecnologías la derivada hacia la televisión social mediante las relaciones con las redes sociales en un intento de interacción con el telespectador tradicional (Caldera, 2014).

En el marco de esos cambios, las televisiones han realizado también algunos importantes para su presencia en la red con difusión de contenidos por vías más cercanas a los nuevos usuarios. Se ofertan nuevos productos y servicios, consiguiendo la ya antigua idea de "televisión a la carta", donde el usuario tiene la posibilidad de decidir qué producto audiovisual quiere consumir, y cuándo lo hace (Caldera y Zapico, 2004).

Las universidades también han conformado sus propias televisiones, de la mano y a la par del desarrollo de las tecnologías. Sin la existencia de la red no habría sido posible el desarrollo de la televisión universitaria.

Una televisión universitaria es aquella que se inscribe dentro de la estructura de una universidad atendiendo a fines no comerciales. Su función es difundir la ciencia y cultura universitaria. Habitualmente están abiertas a la participación de los usuarios, estudiantes y comunidad universitaria en general (Aguadez; Macías, 2008).

La importancia de las televisiones universitarias pasa por la difusión del conocimiento a la sociedad, ya que es el fin de las instituciones universitarias, generar y difundir conocimientos (Aguilera, 1975).

Dichas instituciones se valen de esta herramienta para adaptarse a un nuevo público. La televisión es un medio de comunicación de masas, por lo que se pretende, incorporando este instrumento en las universidades, llegar al mayor número de usuarios posible.

Esto aumenta al incorporar la televisión a las nuevas tecnologías, ya que las televisiones universitarias tienen su presencia en la web, y enlazadas desde la propia página de las universidades. Es aquí donde los usuarios tienen la oportunidad de valerse y participar de ellas. 
Este espacio televisivo debe dedicarse al intercambio informativo, estudio y reflexión de temas vinculados a los problemas de la comunidad universitaria: educación superior, postgrado, investigación científica y desarrollo tecnológico de alto nivel. El contenido debe ir orientado, principalmente, a la educación, aunque puedan estar presentes otro tipo de contenidos, pero siempre sin descuidar el fin esencial de esta herramienta (Cabero, 1988).

\section{OBJETIVOS, HIPÓTESIS Y METODOLOGÍA}

El trabajo que se desarrolla se vertebra con el fin de conseguir alcanzar los objetivos planteados y dar respuesta a las hipótesis de partida. Los objetivos están claramente orientados al conocimiento de la existencia de televisiones educativas en el ámbito universitario español y a identificar los contenidos que se vuelcan en éstas. Por lo tanto, podemos plasmar los objetivos en los siguientes:

a) Identificar la existencia de televisiones universitarias en las instituciones académicas de enseña superior en el ámbito español.

b) Clarificar cuáles son los medios por los cuáles las universidades ponen en marcha las televisiones universitarias.

c) Conocer qué tipo de contenidos son distribuidos por medio de las televisiones universitarias.

d) Mostrar las principales características técnicas y de recuperación de contenidos de las televisiones universitarias españolas.

Las hipótesis planteadas en este trabajo son:

Hipótesis 1: Un alto porcentaje de universidades cuentan con su propia televisión.

Se estima que el desarrollo de las universidades, al igual que el de la televisión educativa, hace prácticamente obligatorio que las universidades cuenten con una televisión para volcar sus contenidos de toda índole, especialmente los relacionados con su actividad educativa. Sin realización de un estudio previo, se estima que al menos un $90 \%$ de las universidades españolas cuentan con una televisión universitaria.

Hipótesis 2: Las televisiones universitarias suelen estar alojadas en las propias web de las universidades.

Por organización de las propias web de las universidades se estima que al menos un $75 \%$ de las universidades alberguen las televisiones universitarias en la URL de la propia institución.

Hipótesis 3: Los contenidos de las televisiones universitarias son en su mayor parte educativos.

Si tenemos en cuenta los objetivos y funciones de las universidades, y por lo tanto el fin último de las televisiones universitarias, estimamos que el cien por cien de las universidades cuentan con contenidos educativos en sus televisiones, lo cual no quita que pudieran contar con otros contenidos distintos.

La metodología que se ha seguido para realizar el trabajo es la siguiente: 
El trabajo desarrollado cuenta con distintas fases en la metodología empleada, todos destinados a la consecución de los objetivos y a la aceptación/refutación de las hipótesis planteadas anteriormente. Todas las fases de la metodología están encaminadas al mejor conocimiento de las televisiones universitarias desde diferentes puntos de vista.

En primer lugar se cuenta con una revisión bibliográfica para llevar a cabo el estado de la cuestión. Se han consultado diferentes bases de datos referenciales y a texto completo para determinar las principales obras a utilizar para desarrollar nuestra labor investigadora.

En un segundo momento, y tras el análisis de dichas obras bibliográficas, se ha realizado la búsqueda de las mejores fuentes de información para la consulta de las televisiones educativas de las universidades españolas, tanto de carácter público como privado.

Posteriormente, se ha desarrollado el trabajo de campo, analizando cada una de las universidades españolas y consultando las web de canales de aquellas que cuentan con una televisión universitaria.

La fuente principal para el acceso a las web de las televisiones ha sido la siguiente url (http://univeridad.es/es/universidades) donde se ha podido localizar un directorio completo de las universidad y su dirección web, tanto de universidades de carácter público como privado, contando con un total de 76 universidades en España.

Este es el listado de Universidades tanto pública como privadas españolas.

\begin{tabular}{|c|c|}
\hline Universitat Abat Oliva CEU & www.uaoceu.es \\
\hline Universitat d'Alicant & www.ua.es \\
\hline Universidad de Alcalá & www.uah.es \\
\hline Universidad Alfonso X el Sabio & www.uax.es \\
\hline Universidad de Almería & www.ual.es \\
\hline Nebrija Universidad & www.nebrija.com \\
\hline $\begin{array}{l}\text { Universitat Autònoma de } \\
\text { Barcelona }\end{array}$ & www.uab.es \\
\hline Universidad Autónoma de Madrid & www.uam.es \\
\hline Universitat de Barcelona & www.ub.edu \\
\hline Universidad de Burgos & www.ubu.es \\
\hline Universidad de Cádiz & www.uca.es \\
\hline Universidad Camilo José Cela & www.ucjc.edu \\
\hline Universidad de Cantabria & www.unican.es \\
\hline Universidad Cardenal Herrera CEU & www.uchceu.es \\
\hline Universidad Carlos III de Madrid & www.uc3m.es \\
\hline Universidad de Castilla-La Mancha & www.uclm.es \\
\hline Universidad Católica de Ávila & www.ucavila.es \\
\hline \multicolumn{2}{|c|}{ Universidad Católica San Antonio de Murcia } \\
\hline \multicolumn{2}{|c|}{ Universidad Católica de Valencia San Vicente M } \\
\hline \multicolumn{2}{|l|}{ Universidad Complutense de Madrid } \\
\hline Universidad de Córdoba & www.uco.es \\
\hline
\end{tabular}




\begin{tabular}{|c|c|}
\hline Universidade da Coruña & aulavirtualtv.uco.es \\
\hline Universidad de Deusto & www.deusto.es \\
\hline Universidad Europea de Madrid & www.uem.es \\
\hline \multicolumn{2}{|c|}{ Universidad Europea Miguel de Cervantes } \\
\hline Universidad de Extremadura & www.unex.es \\
\hline Universidad Francisco de Vitoria & www.ufv.es \\
\hline Universitat de Girona & www.udg.edu \\
\hline Universidad de Granada & www.ugr.es \\
\hline Universidad de Huelva & www.uhu.es \\
\hline IE University & www.ie.edu/university \\
\hline \multicolumn{2}{|c|}{ Universidad Internacional de Andalucía } \\
\hline \multicolumn{2}{|l|}{ Universitat Internacional de Catalunya } \\
\hline \multicolumn{2}{|l|}{ Universidad Internacional de la Rioja } \\
\hline \multicolumn{2}{|c|}{ Universidad Internacional Menéndez Pelayo } \\
\hline Valencian International University & www.viu.es \\
\hline Universidad de Jaén & www.ujaen.es \\
\hline Universitat Jaume I de Castellón & www.uji.es \\
\hline Universidad de la Laguna & www.ull.es \\
\hline Universidad de La Rioja & www.unirioja.es \\
\hline \multicolumn{2}{|c|}{ Universidad de Las Palmas de Gran Canaria } \\
\hline Universidad de León & www.unileon.es \\
\hline Universitat de Lleida & www.udl.es \\
\hline Universidad de Málaga & www.uma.es \\
\hline \multicolumn{2}{|c|}{ Universitas Miguel Hernández de Elche } \\
\hline Mondragon Unibertsitatea & www.mondragon.edu \\
\hline Universidad de Murcia & www.um.es \\
\hline Universidad de Navarra & www.unav.es \\
\hline Universitat Oberta de Catalunya & www.uoc.edu \\
\hline Universidad de Oviedo & www.uniovi.es \\
\hline Universidad Pablo de Olavide & www.upo.es \\
\hline \multicolumn{2}{|c|}{ País VascoEuskal Erriko Unibertsitatea } \\
\hline \multicolumn{2}{|l|}{ Universidad Politécnica de Cartagena } \\
\hline \multicolumn{2}{|l|}{ Universitat Politècnica de Catalunya } \\
\hline Universidad Politécnica de Madrid & www.upm.es \\
\hline $\begin{array}{l}\text { Universidad Politécnica de } \\
\text { Valencia }\end{array}$ & www.upv.es \\
\hline Universitat Pompeu Fabra & www.upf.edu \\
\hline \multicolumn{2}{|c|}{ Universidad Pontificia Comillas (ICAI-ICADE) } \\
\hline \multicolumn{2}{|l|}{ Universidad Pontificia de Salamanca } \\
\hline Universidad Pública de Navarra & www.unavarra.es \\
\hline Universitat Ramón Llull & www.url.edu \\
\hline Universidad Rey Juan Carlos & www.urjc.es \\
\hline Universitat Rovira y Virgil & www.urv.cat \\
\hline Universidad de Salamanca & www.usal.es \\
\hline
\end{tabular}




\begin{tabular}{|c|c|}
\hline Universidad San Jorge & www.usj.es \\
\hline Universidad San Pablo CEU & www.uspceu.com \\
\hline \multicolumn{2}{|c|}{ Universidad de Santiago de Compostela } \\
\hline Universidad de Sevilla & www.us.es \\
\hline \multicolumn{2}{|c|}{ Universidad a distancia de Madrid- Udima } \\
\hline Universitat de les Illes Balears & www.uib.es \\
\hline UNED & www.uned.es \\
\hline Universitat de València & www.uv.es \\
\hline Universidad de Valladolid & www.uva.es \\
\hline Universitat de VIC & www.uvic.cat \\
\hline Universidad de Vigo & www.uvigo.es \\
\hline Universidad de Zaragoza & www.unizar.es \\
\hline
\end{tabular}

Cada uno de estos sitios web ha sido examinado atendido a la tabla de análisis que se desarrolla a continuación:

Universidad:

Naturaleza jurídica: $\quad$ Pública $\quad$ Privada

¿Cuenta con TV Universitaria?

Sí, con presencia en la web

Sí, sin presencia en la No web

\begin{tabular}{|c|c|c|}
\hline ¿Tiene canal en Youtube? & Sí & No \\
\hline \multicolumn{3}{|l|}{ Denominación TV: } \\
\hline \multicolumn{3}{|l|}{ URL: } \\
\hline \multicolumn{3}{|l|}{ Año de creación: } \\
\hline Emisión: & Directo & Diferido \\
\hline \multicolumn{3}{|l|}{$\begin{array}{l}\text { ¿Ofrece vídeos recientes en la página } \\
\text { principal? }\end{array}$} \\
\hline ¿Contiene repositorio de vídeos? & Sí & No \\
\hline \multirow[t]{2}{*}{ Cuenta con: } & Sección destacados & $\begin{array}{l}\text { Motor } \\
\text { búsqueda }\end{array}$ \\
\hline & Directorio/Clasificación & \\
\hline \multirow[t]{2}{*}{ Contenido: } & Informativos & Institucionales \\
\hline & Educativos & Entretenimiento \\
\hline \multicolumn{3}{|l|}{ Divulgativos/culturales } \\
\hline Teléfono: & Sí & No \\
\hline Correo-e: & Sí & No \\
\hline Dirección Postal: & & \\
\hline
\end{tabular}


El cuestionario comienza con los datos identificativos de las universidades: el nombre de la universidad, su naturaleza jurídica y su URL. Al entrar en el sitio web de cada universidad, el nombre de la misma siempre aparece en primer lugar (arriba a la izquierda).

La naturaleza jurídica nos indica si se trata de una universidad pública o privada. Este dato se extrae de los que proporciona la web con el listado de las universidades, al igual que su URL, que nos da el acceso a la página de cada universidad para su análisis.

El siguiente paso es preguntarse si la universidad en cuestión cuenta con una televisión universitaria, y si ésta tiene presencia en la web.

Si la universidad en cuestión no cuenta con una televisión universitaria, el cuestionario termina tras esa pregunta. Si por el contrario tuviera una televisión con presencia en la web, el resto del cuestionario se basa en dicha televisión, siendo el objeto de este estudio.

Aún sin contar con una televisión como tal, las universidades pueden poseer su propio canal en Youtube. Éstos no son exactamente como una televisión, pero sirven para difundir contenidos en forma de vídeos colgados en la red. Al no ser una televisión, a pesar de contar con este tipo de canal, si no tiene además una televisión propia se daría por terminado el análisis de la universidad por medio de la ficha tipo.

En el caso que la universidad posea un canal de televisión se continuará con el resto del cuestionario. Los siguientes apartados hacen referencia a datos de interés general de modo informativo, ya que no tendrán efecto en los resultados que se valorarán en este estudio. Se trata de la denominación de dicho canal de televisión, su URL y su año de inicio de creación, en el caso que ofreciera dicho dato.

A partir de este punto, los datos que se obtengan serán el objeto de estudio, los que se analizarán para obtener los resultados que se extrapolarán a las conclusiones.

En primer lugar se buscará si sus emisiones se realizan en directo, o si por el contrario se emiten en diferido. Por otro lado, nos preguntamos si la página principal ofrece vídeos recientes, y/o si contiene un repositorio de vídeos.

La siguiente sección hace referencia a si la página cuenta con una serie de elementos que son de ayuda a los usuarios a la hora de navegar por la web de la televisión. En este caso se examina si presenta una sección de vídeos destacados, un motor de búsqueda, y un directorio o clasificación de los contenidos.

En otro apartado, lo que se pretende averiguar es qué tipos de contenidos ofrecen los vídeos que se pueden ver en la televisión universitaria: informativos, institucionales, educativos, de entretenimiento, divulgativos o culturales, o de otra índole.

Las tres últimas partes del cuestionario que hemos planteado aluden al modo en el que se puede contactar con la televisión en cuestión. Nos demandamos si ofrece un teléfono, correo electrónico, o una dirección postal. 
Las principales fuentes de información han sido las propias páginas web de las universidades españolas, tanto públicas como privadas, así como su enlace a las cadenas de televisión, ya fueran en su propia sede web o medios de canal de YouTube. Tal y como se puede apreciar se han analizado todas y cada una de las universidades, rastreando y analizando la existencia de televisión universitaria y su presencia web en la distribución de contenidos. Evidentemente se han utilizado obras bibliográficas para definir y contextualizar el trabajo, llegando a redactar por medio de la lectura crítica de los textos un estado de la cuestión en materia de televisión universitaria (López, 1992) (Caldera; Arranz, 2012).

\section{RESULTADO Y ANÁLISIS}

Se van a extraer las principales conclusiones que se infieren a la vista de los datos aportados en los capítulos anteriores. Además, se analizan las hipótesis planteadas en el capítulo inicial, validando o refutando las mismas. Las hipótesis se redactaron una vez tenidas en cuenta las valoraciones establecidas a priori sobre la presencia y contenidos de las televisiones educativas de las universidades públicas y privadas de nuestro país.

Se van a intentar sintetizar las principales ideas extraídas tras el análisis minucioso de los datos conseguidos tras la búsqueda, visita y análisis de las diferentes televisiones universitarias que difunden sus contenidos por medio de la red.

El análisis de dichas web, por lo tanto, ha contado con una doble dimensión: la existencia de televisiones universitarias que cuentan con su plataforma web para la difusión de contenidos o aquellas que han optado por la difusión de sus contenidos por medio de la utilización de la plataforma Youtube como canal de difusión.

\section{CONCLUSIONES}

A continuación exponemos las principales conclusiones:

Destaca la baja cantidad de televisiones universitarias en España, ya que tan sólo el $50 \%$ de las universidades apuestan por esta herramienta como importante apoyo a la difusión de conocimientos y refuerzo de la educación. El 62 \% de las universidades públicas españolas cuentan con televisión, mientras que en el caso de las televisiones privadas se trata tan sólo del $27 \%$.

\section{Universidades Españolas}

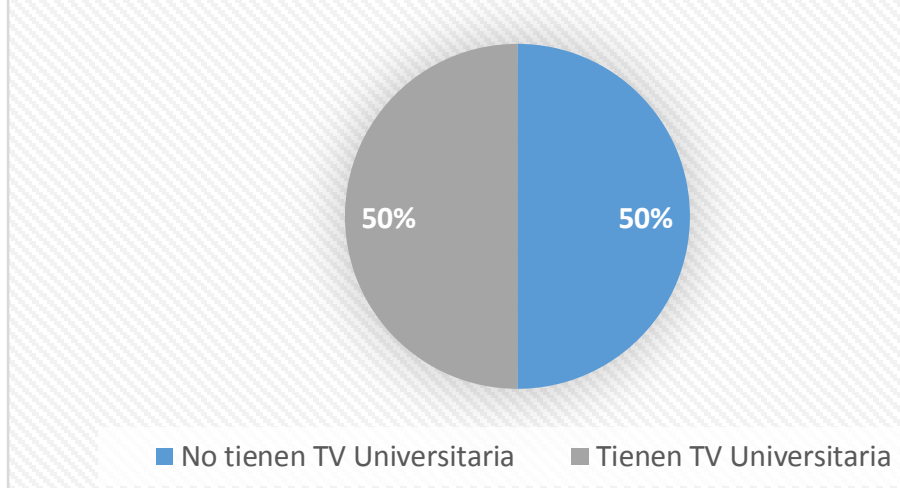


Es destacable igualmente el alto porcentaje de universidades que presentan un canal en el portal Youtube que requiere menos medios para su implantación y gestión, pero que a su vez ofrece menos posibilidades que las televisiones en una sede web convencional. Se trata de un $84 \%$ del número total de universidades.

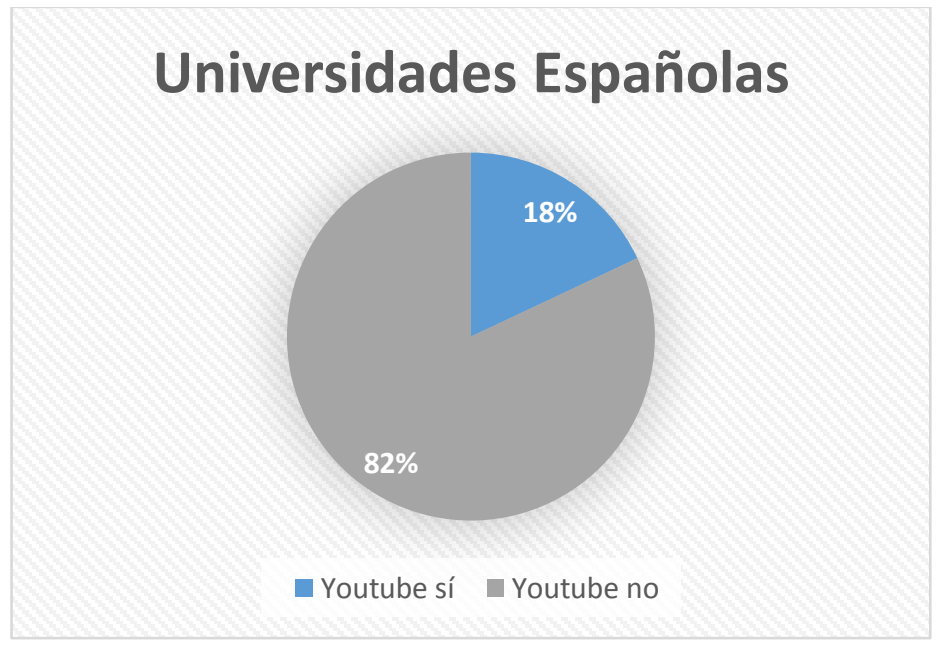

El $50 \%$ de las televisiones universitarias cuentan con emisión en directo. Debe quedar aquí claramente reflejado que esto no se traduce en una emisión constante durante las 24 horas, ni tan siquiera el hecho de contar con la parrilla de programación estable.

En el $50 \%$ destacado queremos señalar que dichas emisiones en directo se realizan en las cadenas, de forma regular o esporádica. Advertir que ese $50 \%$ de televisiones universitarias con emisión en directo son únicamente públicas, ya que ninguna de las universidades privadas apuesta por emitir contenidos en directo. En el caso de las emisiones en diferido se trata de un $85 \%$ de las televisiones las que realizan dichas emisiones, siendo equitativas en universidades públicas y privadas.

De acuerdo a los aspectos formales, tanto las televisiones públicas como privadas ofrecen, en porcentajes considerablemente altos, elementos de importancia para un fácil manejo de la web televisiva como son: vídeos recientes, vídeos destacados, motor de búsqueda, directorio de vídeos o repositorio de vídeos.

En las televisiones educativas de universidades privadas los porcentajes de contenidos atendiendo a su temática no varían entre sí de forma significativa, con excepción de los contenidos educativos e institucionales que cuentan con un mayor número de horas en la difusión de contenidos. Es bastante lógico una vez entendida la naturaleza, fines y objetivos de una televisión universitaria.

No obstante, llama poderosamente la atención que un $29 \%$ de las televisiones universitarias de carácter privado no cuenten con información educativa, lo que se traduce en una utilización de la televisión con fines de marketing básicamente, no centrándose en lo que entendemos debería ser una prioridad para las televisiones universitarias: la ayuda o complemento a la docencia y a la investigación. Es destacable, y sostiene lo defendido anteriormente, que el $71 \%$ de las cadenas cuentan con información institucional. 
Los contenidos informativos, de entretenimiento y divulgativos/culturales aparecen en un $57 \%$ de las televisiones universitarias analizadas.
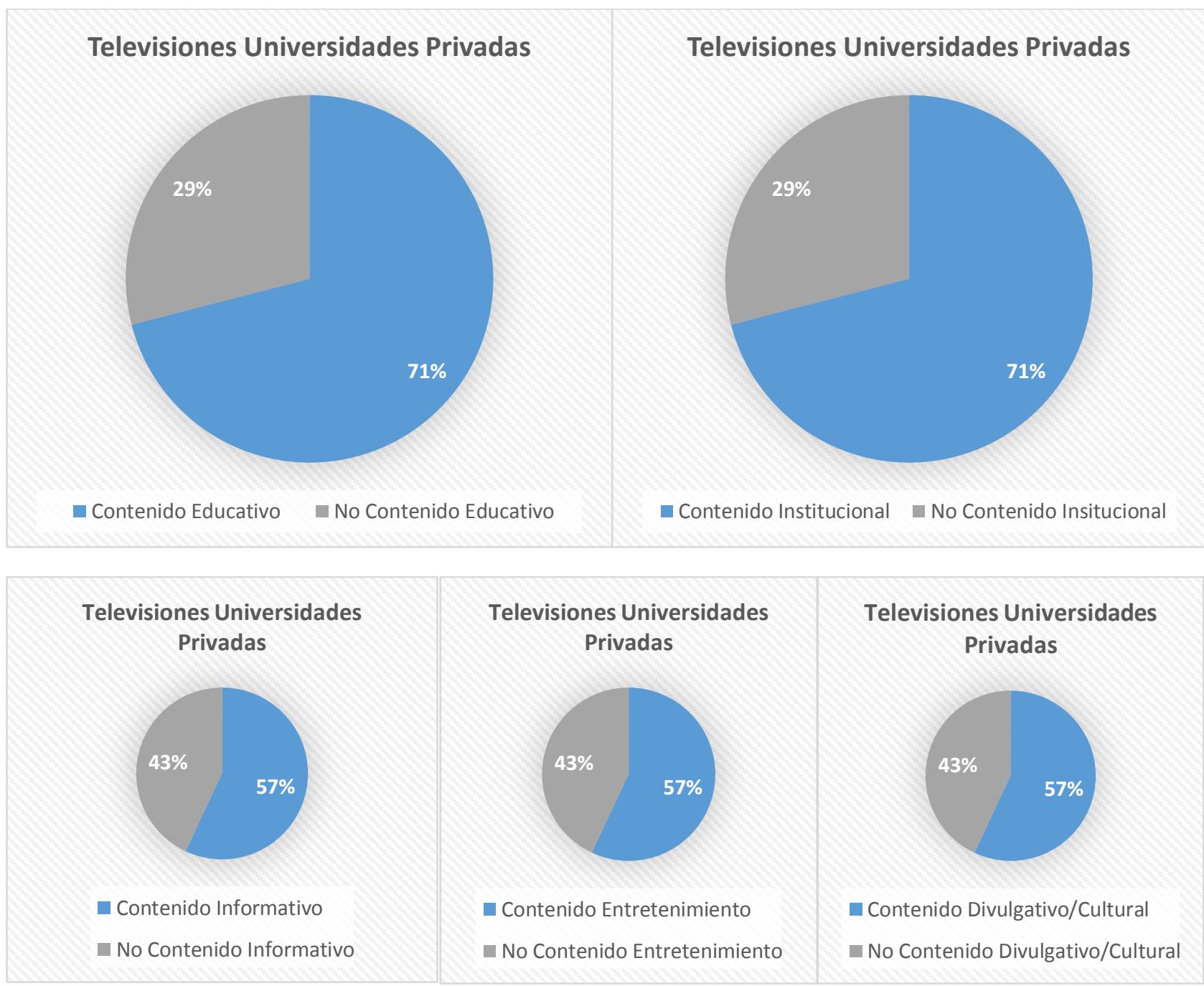

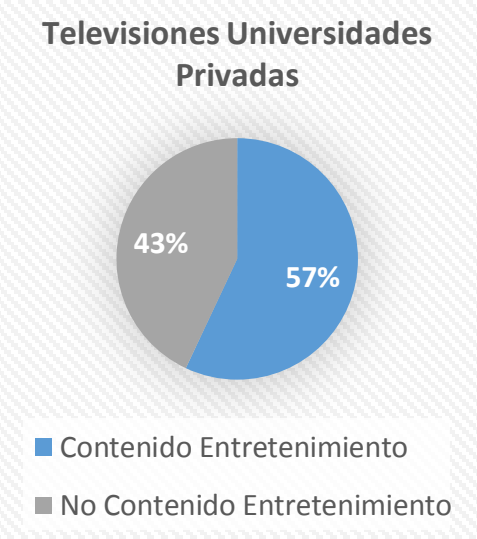

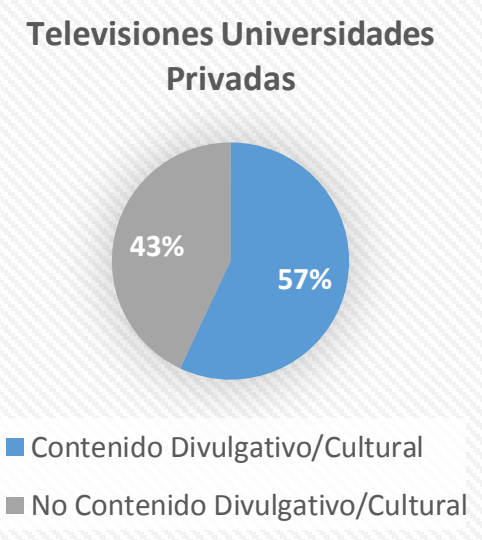

\section{Privadas}

En las televisiones públicas la diferencia entre los porcentajes de presencia en las web de los distintos contenidos es más dispar que en las privadas. Sobresale por mucho, entre los otros tipos de contenidos, el 94\% de universidades públicas que cuentan con contenidos educativos en sus televisiones, siendo lo más apropiado ya que éstos deben ser la base de la televisión educativa. También puede considerarse alto el porcentaje de televisiones universitarias públicas en España con contenidos institucionales, sin tener en cuenta otros contenidos, con mucha diferencia respecto al resto de contenidos, siendo algo normal por la importancia de presentar los documentos audiovisuales de los contenidos propios de la institución. En el caso de los contenidos informativos, de entretenimiento y divulgativos/culturales los porcentajes son: $52 \%$, $42 \%$ y $39 \%$ respectivamente. 


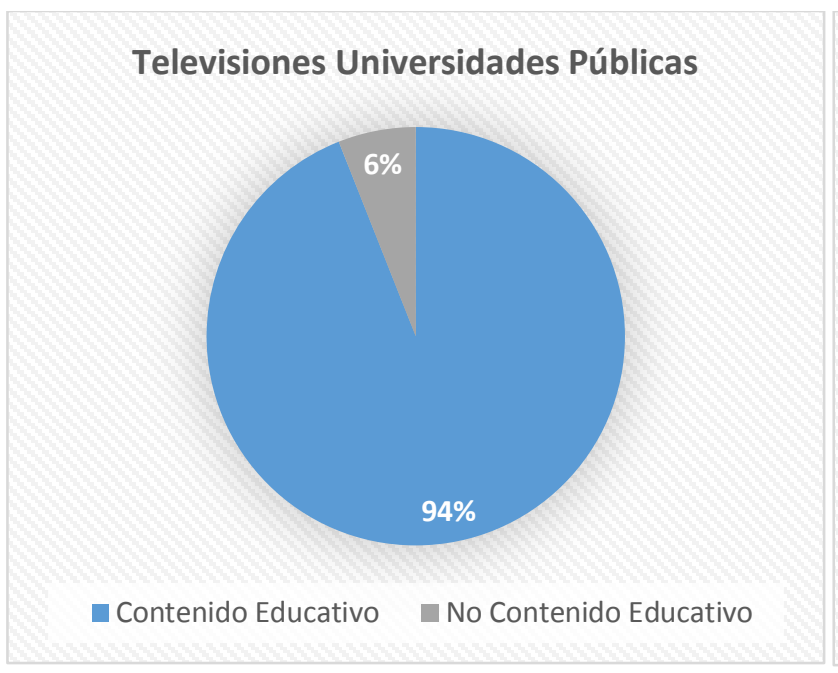

\section{Televisiones Universidades Públicas}
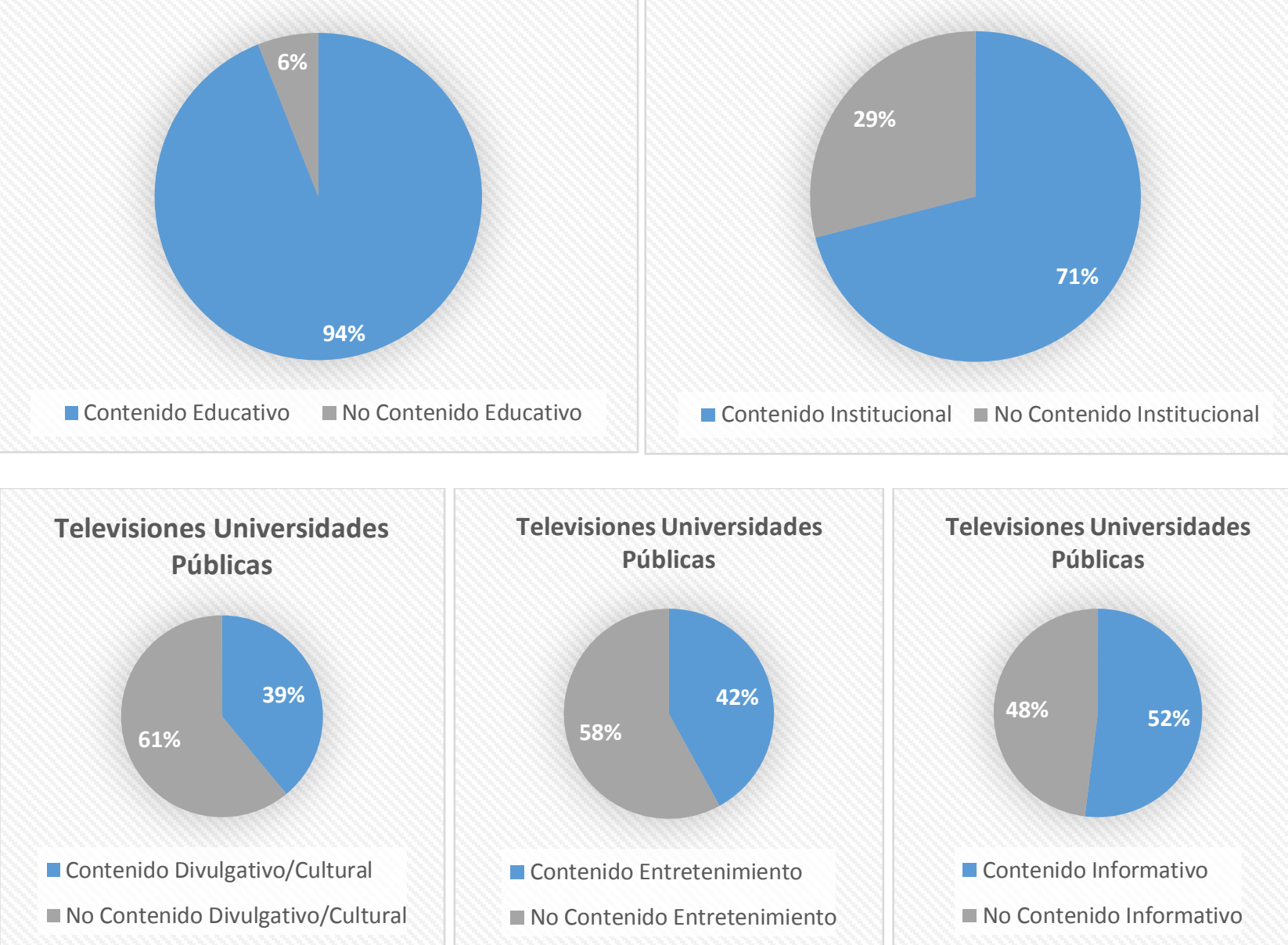
Televisiones Universidades
Públicas

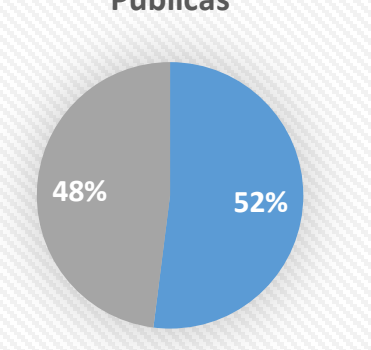

- Contenido Informativo

No Contenido Informativo

El acceso a las plataformas web de las televisiones universitarias españolas se realiza por medio de hiperenlaces a otras web con el mismo entorno institucional o a canales televisivos incluidos en Youtube. Lo más destacable es que dicho acceso no está siempre en la página principal de las sedes web, ni tan siquiera destacado en aquellos casos con los que cuentan con dicha realidad. En definitiva, no se aporta información útil y ágil de estas televisiones, siendo necesario escalar en la web, en algunos casos, varios niveles hasta localizar dicha televisión universitaria. Desde el punto de vista ergonómico, las televisiones universitarias cuentan con un dispar tratamiento, ya que mientras unas incorporan los mismos atributos físicos que su web matriz, otras son elaboradas de forma independiente.

Para finalizar este apartado, se quisiera analizar las hipótesis iniciales.

Hipótesis 1: Un alto porcentaje de universidades cuentan con su propia televisión

Se refuta la primera hipótesis, ya que tan sólo el $50 \%$ de las universidades españolas cuentan con una televisión educativa donde volcar sus contenidos para su difusión, y no un $90 \%$ como se indicaba en la hipótesis. 
Por lo tanto, podemos determinar que existe un número escaso de televisiones universitarias en nuestro país, siendo las televisiones privadas aquellas que cuentan con un déficit porcentualmente más significativo.

\section{Hipótesis REFUTADA}

Hipótesis 2: Las televisiones universitarias suelen estar alojadas en las propias web de las universidades.

Todas las universidades españolas que cuentan con televisión tienen un enlace a la misma en la propia web de la universidad, aunque en ocasiones de manera muy rebuscada para los usuarios, siendo más útil que se encuentre a simple vista en un buen lugar en la página principal.

\section{Hipótesis VALIDADA}

Hipótesis 3: Los contenidos de las televisiones educativas son en su mayor parte educativos.

El 89\% de las televisiones analizadas de las universidades españolas ofrecen contenidos educativos. Estos contenidos deberían ser el principal motivo de creación de dichas televisiones.

Aunque se observe un altísimo porcentaje, se valida parcialmente la hipótesis ya que se esperaba un $100 \%$ de televisiones universitarias con contenidos educativos, independientemente de ofrecer contenidos de otra índole.

En el caso de las universidades públicas, proporcionalmente presentan un porcentaje cercano al esperado, un $94 \%$, sin embargo, tan sólo un $71 \%$ de las universidades privadas ofrecen contenidos educativos, por lo que están lejos del objetivo principal de una televisión educativa.

Hipótesis VALIDADA PARCIALMENTE.

\section{CONCLUSIONES}

La televisión es un fenómeno utilizado con fines de lo más variados. Utilizado desde el Poder para generar opinión, para contra informar, para vender, para entretener, y por qué no, también para educar. Y dentro de las muchas potencialidades que ofrece la televisión como instrumento transmisor de información, las universidades también han visto en este medio una manera de comunicarse con su comunidad, además de ser una fórmula útil de conexión con la sociedad a la cual sirve.

Tal y como se ha podido ver en el trabajo, aunque dicha potencialidad ya existe desde hace algunos años, existe una cierta resistencia por parte de algunas universidades, especialmente las privadas, para la implementación de una televisión universitaria. Dicha televisión universitaria no siempre cuenta con un uso adecuado por parte de la institución académica, sirviendo en muchos casos para la venta de marca y de la imagen de la institución.

No quedan muy bien paradas en esta foto fija las universidades privadas, las cuales no llegan a facilitar información educativa, en algunos de los casos. Mejor posición tienen las universidades públicas españolas que sí parecen utilizar de forma más o menos correcta dicha televisión, si de forma correcta entendemos como plataforma de difusión de contenidos educativos. 
No obstante, el uso e importancia que se le otorga a las televisiones universitarias aún está en desarrollo. No se utiliza toda su potencialidad ya que en muchos casos contamos con una serie de repositorios de vídeos que están presentes en un canal de YouTube. Falta organización, sistematización en la difusión de los contenidos, falta en definitiva una apuesta clara por el método televisivo como herramienta de difusión. Y decimos el método televisivo ya que cada vez los medios audiovisuales están más presentes como método de difusión de contenidos, como herramienta pedagógica con un indudable valor y de mayor facilidad de asimilación por parte de los alumnos universitarios. Se utilizan otras herramientas, otras fórmulas de difusión de contenidos audiovisuales como las plataformas de e-learning, pero siempre con una difusión mucho más concreta, local, centrado muchas veces en la propia aula.

Para terminar, señalar el gran potencial aún sin desarrollar de las televisiones universitarias. Y aunque existen intentos muy desarrollados, la generalidad de las universidades analizadas en este trabajo nos muestra aún muchas televisiones en desarrollo y otras muchas en las que aún se están buscando unas políticas claras de uso.

En España aún queda mucho camino por recorrer para llegar a que la totalidad de las universidades españolas, tanto públicas como privadas, presenten una televisión universitaria y, lo que es más importante, que éstas cumplan los objetivos de una televisión educativa. Es muy importante que ofrezcan una web con los elementos y contenidos necesarios para su fácil usabilidad y entendimiento para los usuarios, y que éstos puedan servirse de dicha herramienta como apoyo a sus estudios.

Hay que prestar mucha atención a la hora de crear la web no sólo a sus contenidos (base fundamental), sino también a aspectos como la orientación al usuario para moverse por ella, para buscar contenidos más rápidamente, dónde colocar cada elemento dentro de la página, etc., así como la ubicación y el modo de señalar el enlace a la televisión universitaria dentro de la web de la propia institución.

Así mismo habría que destacar la falta de difusión que presentan las televisiones educativas, ya que la mayoría de los estudiantes ni tan siquiera son conscientes de su existencia.

\section{RECONOCIMIENTOS}

El presente trabajo fue patrocinado por el Proyecto Prometeo de la Secretaría de Educación Superior, Ciencia, Tecnología e Innovación de la República del Ecuador.

Este trabajo ha sido financiado por el Gobierno de Extremadura (Consejería de Educación, Ciencia y Tecnología) y el Fondo Social Europeo dentro del plan de apoyo a las actuaciones de los Grupos de Investigación inscritos en el catálogo de la Junta de Extremadura. GR10019.

\section{BIBLIOGRAFÍA}

AGUADED Gómez, J. Ignacio; MACÍAS GÓMEZ, Yolanda. "Televisión universitaria y servicio público". Comunicar. Huelva, 2008, v. XVI, no 31, p. 681-689.

AGUILERA GAMONEDA, Joaquín de. Dimensión y sistema de la televisión educativa. Madrid: Editorial Nacional, 1975. 
CABERO ALMENARA, Julio [et. al.]. La televisión educativa iberoamericana: Evaluación de una experiencia. Sevilla: [ATEI], D.L. 1998.

CALDERA-SERRANO, J (2014). Resumiendo documentos audiovisuales televisivos: propuesta metodológica. Perspectivas em Ciencia da Informaçao, vol. 19, núm. 2, pp. 147-158

CALDERA-SERRANO, J; ARRANZ-ESCACHA, P (2012). Documentación audiovisual en televisión. Barcelona: UOC. ISBN 9788-84-9029-982-1

CALDERA-SERRANO, J; ZAPICO-ALONSO, F (2004). La fórmula de comunicación de Lasswell como método para implementar bases de datos documentales en los medios audiovisuales. Investigación Bibliotecológica, vol. 18, núm. 37, pp.110-131.

LÓPEZ YEPES, Alfonso. Manual de documentación audiovisual. Pamplona: Universidad de Navarra, 1992. 